\title{
Smartphone applications for chronic pain management: a critical appraisal
}

\section{John C Alexander Girish P Joshi}

Department of Anesthesiology \& Pain Management, University of Texas Southwestern Medical Center, Dallas, TX, USA
Correspondence: John C Alexander Department of Anesthesiology \& Pain Management, University of Texas

Southwestern Medical Center, 5323 Harry Hines Boulevard, Dallas, TX 75390-9068, USA

Email john.alexander@utsouthwestern.edu
This article was published in the following Dove Press journal: Journal of Pain Research

26 September 2016

Number of times this article has been viewed

Chronic pain is a common condition with significant detrimental physical, psychological, social, and economic impact. The Institute of Medicine estimates that $>100$ million Americans suffer from chronic pain, ${ }^{1}$ representing approximately one-third of the entire population of the US. ${ }^{2}$ Conservative estimates suggest that well over US $\$ 500$ billion per year is spent in the treatment of these pain conditions, not to mention the lost productivity of these individuals or the burden that their suffering engenders for patients and their families. Despite tremendous efforts, chronic pain continues to be a major societal problem. ${ }^{1}$

Smartphones have become one of the most rapidly adopted technologies in the modern history of mankind allowing for previously unimaginable opportunities for communication and access to information. ${ }^{3}$ Powering this societal revolution is not so much the onboard or attachable hardware for smartphones, but the dizzying array of software programs that use the hardware to add novel functions; we call the unifying software programs "applications" or, more commonly, "apps". While each phone comes with onboard technologies, such as Wi-Fi, Bluetooth, lights, microphones, cameras, accelerometers, and even barometers, it is the development of the app that combines these functionalities to create new and innovative uses for the same hardware for everything from Skype ${ }^{4}$ to Pokémon GO. ${ }^{5}$

The use of such applications within the health care industry continues to grow, and it is estimated that the market for mobile health apps will grow to US\$26 billion in 2017. ${ }^{6}$ The immense size of this market is due to the functional flexibility that apps can provide. These emerging technologies also provide new opportunities to engage with patients and improve health care outcomes. Studies have shown that mobile phone messaging helps to improve patient engagement outside the clinic or hospital and facilitates self-management of chronic diseases such as hypertension, diabetes, and asthma, ${ }^{7}$ and app-based systems may also be of use in this regard. While the overall market for apps in health care is robust, the market segment for pain management is less than impressive.

It is clear that pain is a complex phenomenon and thus requires a multifaceted and multidisciplinary approach to achieve optimal outcomes. Of the several pain management approaches, the biopsychosocial components to the pain experience have been shown to be the most effective means of treating chronic pain, yet they are underutilized. ${ }^{8}$ New technologies such as the smartphone apps that have come 
into existence may help close the gap in utilization of these evidence-based treatments. In considering the current and potential market for pain management apps, one must take into account that some apps should be targeted to patients, while others should be targeted to health care professionals. The most basic provider-focused apps would simply serve as a reference giving factual information on variety of topics. Such apps could be used to enhance disease-specific knowledge and provide evidence-based treatment options including their limitations. A layer of functionality can also be added where decision support tools are added to improve adherence to evidence-based clinical practice guidelines. ${ }^{9}$

Patient-facing apps could provide the most benefits. Such apps would seek to engage patients in between (or perhaps instead of) traditional outpatient visits and empower them to take a more active role in the management of their ailment, to improve pain relief, to facilitate functioning, and to return to daily living. Thus, pain management apps should integrate biological processes, psychological factors, and sociocultural factors. Strategies to cope with symptoms (eg, cognitive behavioral therapies) and engage in social support are needed to promote optimal outcomes.

Electronic diaries have been shown to improve compliance and decrease the risk of recall bias compared with the standard retrospective pain assessment performed in clinical encounters. ${ }^{10}$ Point-of-care social support apps would be particularly useful as strong peer relationships are predictive of positive judgments of independence, emotional adjustment, and identity formation. Self-management involves interaction of health behaviors and related processes that patients and families engage in to care for a chronic condition. Self-management apps seek to intervene to provide the ability to manage the multidimensional aspects of chronic pain. In addition, comprehensive interactive face-to-face psychoeducational interventions lead to symptom reduction and improved health-related quality of life compared with care that is strictly medication focused, with a postulated mechanism being enhanced self-efficacy and empowerment over disease and symptom management. Compliance with such face-to-face encounters can be difficult to perform on a routine basis due to cost and time constraints, but remote delivery of such services has been shown to improve outcomes in pediatric chronic pain populations. ${ }^{11}$ If these types of apps can drive better outcomes for chronic diseases without requiring face-to-face patient interactions, then they may become a valuable means of driving down the overall cost of patient care. ${ }^{12}$ Recent surveys show that while only $10 \%$ of patients utilize such virtual care services, $>60 \%$ would be open to utilizing virtual care services through these technologies, which may help improve patient engagement outside the clinic or hospital. ${ }^{13}$

Several studies have attempted to ascertain both the quantity and quality of apps marketed to improve chronic pain. ${ }^{14-16}$ The conclusions of the authors are remarkably similar over time. First, the vast majority of apps did not follow evidence-based guidelines, thus calling the function of the app into question. This is likely related to the second common conclusion of these studies that health care providers were rarely involved in the development of these apps. If clinicians are not leveraging their expertise into the development of such apps, then it is unsurprising that the content is not clinically useful. The final common critique is that, unlike in the pharmaceutical or medical device industries, there is a lack of peer-reviewed validation studies for the purported functionalities of commercially available applications. This is not a problem unique to the pain management app market segment, though.

Smartphone-based health care apps have been largely passed over by the US Food and Drug Administration (FDA) with regard to regulation due in part to a desire to allow innovation to flourish. ${ }^{17}$ It is unlikely that the FDA will ever take a leading role in the regulation of this market. First and foremost, it is not within the FDA's jurisdiction to regulate most of the apps that are available to consumers. In 2015, the FDA published a statement to clarify its position on the status of what it terms "mobile medical apps" and further defines them as mobile device software applications intended to be used as a medical device or to transform a platform into a medical device but further refined its position stating that it intends to exercise its "regulatory oversight only to those mobile medical apps that are medical devices and whose functionality could pose a risk to patient safety". For instance, the FDA lists the sorts of apps for which it intends to "exercise enforcement discretion" (ie, not yet require FDA approval). These include mobile medical apps that provide tracking or organizing functions of health care data, routine clinical calculators, or access to reference data. The focus of the FDA's regulatory oversight is for those mobile medical apps that "use a sensor or electrode attached to the mobile platform or tools within the mobile platform itself" for specific functions that could pose a risk to patient safety if a malfunction were to occur. ${ }^{18}$ Second, there are simply too many apps to regulate. The ease with which applications can be created outstrips the ability of any agency to adequately regulate this industry. By some estimates, there may be $>165,000$ health care-related apps available to consumers. ${ }^{19}$ 
This lack of regulation leaves validation in the hands of the medical community. While there are some small studies attempting to validate some of the more popular health care smartphone apps, ${ }^{20,21}$ such studies are lacking in the pain management market segment.

These critiques, though, give a blueprint for a means by which those of us who care for patients with chronic pain may help develop and validate apps for clinical use. In the same way that health care providers would likely be unable to create usable apps, the evidence seems to indicate that developers are not able to make clinically relevant apps. The collaboration of developers with health care providers, though, will help to ensure that apps are both usable by consumers and contain clinically valid data utilizing evidence-based guidelines. Another critique is the lack of validation of apps in peer-reviewed literature. There are several recent examples where protocols or development strategies for proposed pain apps were published for peer-review, ${ }^{22-24}$ but validation studies of completed pain management apps remain the exception rather than the rule. ${ }^{10}$ Thus, while we see the possible utility of smartphone apps in pursuit of the goal of improved engagement with pain patients leading to better outcomes, much work remains to be done to ensure such apps are developed with a strong basis in evidence-based guidelines and validated through robust, peer-reviewed studies.

\section{Disclosure}

The authors report no conflicts of interest in this work.

\section{References}

1. Institute of Medicine of the National Academies. Relieving Pain in America: A Blueprint for Transforming Prevention, Care, Education, and Research. Washington, DC: National Academies Press (US); 2011.

2. United States Census Bureau. Vintage 2011: National Tables. Available from: http://www.census.gov/popest/data/historical/2010s/vintage_2011/. Accessed August 9, 2016.

3. Flurry Insights. iOs and Android Adoption Explodes Internally. 2016. Available from: http://flurrymobile.tumblr.com/post/113379358945/ ios-and-android-adoption-explodes-internationally. Accessed April 27, 2016.

4. Skype Communications S.a.r.l. Skype-for-iPhone. Available from: https://itunes.apple.com/us/app/skype-for-iphone/id304878510?mt=8. Accessed August 9, 2016

5. Niantic, Inc. Pokémon GO. Available from: https://itunes.apple.com/ us/app/pokemon-go/id1094591345?mt=8. Accessed August 1, 2016

6. Research2guidance. Mobile Health Market Report 2013-2017. 2013. Available from: http://research2guidance.com/product/mobile-healthmarket-report-2013-2017/. Accessed August 9, 2016.
7. McEvoy MD, Hand WR, Stiegler MP, et al. A Smartphone-based Decision Support Tool Improves Test Performance Concerning Application of the Guidelines for Managing Regional Anesthesia in the Patient Receiving Antithrombotic or Thrombolytic Therapy. Anesthesiology. 2016;124: 186-198.

8. Schatman ME. Interdisciplinary chronic pain management: international perspectives. Pain. 2012;20(7):1e-5e.

9. de Jongh T, Gurol-Urganci I, Vodopivec-Jamsek V, Car J, Atun R. Mobile phone messaging for facilitating self-management of long-term illnesses. Cochrane Database Syst Rev. 2012;2(12):CD007459.

10. Vardeh D, Edwards RR, Jamison RN, Eccleston C. There's an App for That: Mobile Technology Is a New Advantage in Managing Chronic Pain. Pain. 2013;XXI(6):1-7.

11. Fisher E, Law E, Palermo T, Eccleston C. Psychological therapies (remotely delivered) for the management of chronic and recurrent pain in children and adolescents. Cochrane Database Syst Rev. 2015;3(3): CD011118.

12. CMS. National Health Expenditures 2014 Highlights. Available from: https://www.cms.gov/Research-Statistics-Data-and-Systems/StatisticsTrends-and-Reports/NationalHealthExpendData/Downloads/highlights. pdf. Accessed August 9, 2016.

13. Industry Research: Healthcare. 2016 Connected Patient Report: Insights into patient preferences on telemedicine, wearables and post-discharge care. Available from: https://secure2.sfdcstatic.com/assets/pdf/industries/2016state-of-the-connected-patient.pdf. Accessed August 9, 2016.

14. Lalloo C, Jibb LA, Rivera J, Agarwal A, Stinson JN. "There's a Pain App for That": Review of Patient-targeted Smartphone Applications for Pain Management. Clin J Pain. 2015;31:557-563.

15. Reynoldson C, Stones C, Allsop M, et al. Assessing the quality and usability of smartphone apps for pain self-management. Pain Med.2014; 15:893-909.

16. Rosser BA, Eccleston C. Smartphone applications for pain management. J Telemed Telecare. 2011;17:308-312.

17. Alexander JC, Joshi GP. Smartphone Application-based Medical Devices: Twenty-first Century Data Democratization or Anarchy? Anesth Analg. Epub 2016 Aug 22.

18. Mobile Medical Applications: Guidance for Industry and Food \& Drug Administration Staff. Available from: http://www.fda.gov/downloads/ MedicalDevices/...UCM263366.pdf. Accessed August 9, 2016.

19. S Misra. New report finds more than 165,000 mobile health apps now available, takes close look at characteristics \& use; 2015. Available from: http://www.imedicalapps.com/2015/09/ims-health-apps-report/\#. Accessed August 9, 2016.

20. Alexander JC, Minhajuddin A, Joshi GP. Comparison of smartphone application-based vital sign monitors without external hardware versus those used in clinical practice: a prospective trial. J Clin Monit Comput. Epub 2016 May 12.

21. Plante TB, Urrea B, MacFarlane ZT, et al. Validation of the Instant Blood Pressure Smartphone App. JAMA Intern Med. 2016;176:700-702.

22. Blodt S, Pach D, Roll S, Witt CM. Effectiveness of app-based relaxation for patients with chronic low back pain (Relaxback) and chronic neck pain (Relaxneck): study protocol for two randomized pragmatic trials. Trials. 2014;15:490.

23. de la Vega R, Roset R, Castarlenas E, Sanchez-Rodriguez E, Sole E, Miro J. Development and testing of painometer: a smartphone app to assess pain intensity. $J$ Pain. 2014;15:1001-1007.

24. Stinson JN, Lalloo C, Harris L, et al. iCanCope with Pain ${ }^{\mathrm{TM}}$ : User-centered design of a web- and mobile-based self-management program for youth with chronic pain based on identified health care needs. Pain Res Manag. 2014;19:257-265. 
Dove Medical Press encourages responsible, free and frank academic debate. The content of the Journal of Pain Research 'Editorial' section does not necessarily represent the views of Dove Medical Press, its officers, agents, employees, related entities or the Journal of Pain Research editors. While all reasonable steps have been taken to confirm the content of each Editorial, Dove Medical Press accepts no liability in respect of the content of any Editorial, nor is it responsible for the content and accuracy of any Editorial.

Journal of Pain Research

Publish your work in this journal

The Journal of Pain Research is an international, peer reviewed, open access, online journal that welcomes laboratory and clinical findings in the fields of pain research and the prevention and management of pain. Original research, reviews, symposium reports, hypothesis formation and commentaries are all considered for publication. 